\title{
Detection of symmetry in tachistoscopically presented dot patterns: Effects of multiple axes and skewing
}

\author{
JOHAN WAGEMANS, LUC VAN GOOL, and GÉRY D'YDEWALLE \\ University of Leuven, Leuven, Belgium
}

\begin{abstract}
We examined the effects of multiple axes and skewing on the detectability of symmetry in tachistoscopically presented (100-msec) dot patterns to test the role of normal grouping processes based on higher order regularities in element positions. In addition to the first-order regularities of orientational uniformity and midpoint collinearity (Jenkins, 1983), bilateral symmetry (BS) gives rise to second-order relations between two pairs of symmetric elements (represented by correlation quadrangles). We suggest that they allow the regularity (i.e., BS) to emerge simply as a result of the position-based grouping that takes place normally, so that no special symmetrydetection mechanism has to be postulated. In combination with previously investigated variablesnumber and orientation of axes-we introduced skewing (resulting from orthographic projection of BS) to manipulate the kind and number of higher order regularities. In agreement with our predictions, the data show that the effect of skewing angle (varied at three $15^{\circ}$ steps, clockwise and counterclockwise) on the preattentive detectability of symmetry (measured with $d^{\prime}$ ) increases as the number of axes decreases. On the basis of some more specific findings, we suggest that it is not as much the number of correlation quadrangles that determines the saliency of a regularity as it is the degree to which they facilitate or "bootstrap" each other.
\end{abstract}

During the time since Mach's (1886/1959) observations on the special status of bilateral or mirror symmetry for the human perceptual system, numerous experiments have been done on its detection and use. With respect to the latter, abundant data indicate the influence of symmetry on several perceptual and cognitive processes, such as scanning, encoding, short-term memory, recognition, representation, imagination, the establishment of a reference frame, and judgments of numerosity, complexity and goodness, or aesthetic value (see, e.g., Chipman \& Mendelsohn, 1979; Fox, 1975; Howe \& Jung, 1987; Locher \& Nodine, 1973, 1989; Palmer, 1985; Pashler, 1990; Szilagyi \& Baird, 1977; Yodogawa, 1982). All this research suggests that symmetry is a very salient visual property that the human visual system must detect efficiently and rapidly.

The authors are supported by grants from the Belgian National Fund for Scientific Research (NFWO) to J. Wagemans and from the Belgian Institute for the Encouragement of Scientific Research in Industry and Agriculture (IWONL) to L. Van Gool. The interdisciplinary collaboration is made possible by grants from the University of Leuven (OT/89/11) and from the Beigian Fund for Joint Basic Research (FKFO). The equipment used is sponsored by the Belgian Ministry of Science (AI-Impulse Program) and the University of Leuven (GOA). We gratefully acknowledge the technical support of $\mathrm{N}$. Bovens, D. Christiaens, R. Delabastita, M. Devijver, M. Lenaerts, and J. Van Rensbergen. We are indebted to D. Foster, R. Fox, P. Locher, and some anonymous reviewers for comments on a previous draft. J. Wagemans and G. d'Ydewalle are associated with the university's Department of Experimental Psychology; L. Van Gool is in the Department of Electronics. Correspondence should be sent to Johan Wagemans, Laboratory of Experimental Psychology, University of Leuven, Tiensestraat 102, B-3000 Leuven, Belgium (e-mail address: fpaas10@blekul11.bitnet).
Although the use of signal-detection measures (e.g., $d^{\prime}$ ) and tachistoscopic presentation (e.g., 25 or $100 \mathrm{msec}$ ) has shown that human perceivers are indeed capable of detecting bilateral symmetry (BS; see, e.g., Barlow \& Reeves, 1979; Carmody, Nodine, \& Locher, 1977), several decades of experimentation and modeling have not succeeded in clarifying how we do it exactly. To be sure, the effects of several different variables on the efficiency and speed of symmetry detection have been studied extensively. Naturally, this kind of research has yielded important information about the general principles of the mechanism and the constraints within which it operates. In addition, the implications of these findings have been accounted for in some preliminary accounts of symmetry detection. Nevertheless, several essential questions remain unanswered. We will address them by discussing examples from experimental and theoretical work. Our own research fits into the same tradition, in that we are trying to find out the effects of particular variables (here, multiple axes and skewing) on symmetry detection, but we are attempting to go further, in that our research is more explicitly focused on testing a particular proposal about a mechanism of symmetry detection.

\section{EFFECTS ON SYMMETRY DETECTION: PRELIMINARY ACCOUNTS}

\section{Axis Orientation}

The variable most often studied in research on detection of symmetry is the orientation of the axis of symmetry. Whereas most studies (e.g., Fisher \& Bornstein, 
1982; Jenkins, 1985) have shown that symmetry about a vertical (V) axis is most salient, and hence easier to detect, as measured by accuracy and response times (RTs), there is some controversy about the relative detectability of horizontal $(\mathrm{H})$ and oblique $(\mathrm{O})$ symmetry. Palmer and Hemenway (1978) have explained the difference between their results regarding orientational effects, indicating an oblique effect ( $\mathrm{RT}_{\mathbf{V}}<\mathrm{RT}_{\mathbf{H}}<\mathrm{RT}_{\mathrm{O}}$ ), and Corballis and Roldan's (1975), indicating a mental rotation effect $\left(\mathrm{RT}_{\mathrm{V}}<\mathrm{RT}_{\mathrm{O}}<\mathrm{RT} \mathrm{T}_{\mathrm{H}}\right.$ ), by the absence or presence, respectively, of an explicitly drawn axis of symmetry. In addition, this has been interpreted as evidence for a stage in which a potential axis is selected prior to the evaluation of symmetry about it and for the bias of this stage toward V. Recently, Pashler (1990) has come to a similar conclusion on the basis of data from two experimental paradigms: response times with extended exposure duration and accuracy with tachistoscopic presentation (i.e., $100 \mathrm{msec}$ ).

Palmer and Hemenway (1978, Experiment 1) found considerably higher detectability of double and quadruple symmetry (i.e., resulting from a reflection of a pattern about two and four axes, respectively). This finding led them to suggest that the first stage of axis selection follows a variable order. On the average, a symmetry axis will be selected sooner when there are multiple axes from which to choose. In their Experiment 2, Palmer and Hemenway instructed the subjects to detect $\mathrm{V}$ symmetry only, in order to eliminate the selection process. This manipulation resulted in a considerable reduction in RTs for all symmetries, yet the advantage of the multiple symmetry remained. This result is somewhat surprising, if the variable selection is indeed the only explanation for the multiple symmetry advantage. It has been interpreted, therefore, as a facilitation of the evaluation stage on the basis of the good gestalt of the pattern halves that have to be compared.

In summary, then, Palmer and Hemenway (1978, p. 700) have suggested the following dual process model with which to interpret their results.

First, the observer selects a potential axis by a crude, but rapid, analysis of symmetry in all orientations simultaneously.... This selection process is biased toward $V$ and, to a lesser extent, $\mathrm{H}$ axes rather than $\mathrm{O}$ ones. The actual order of selection is assumed to be variable rather than fixed. If a given axis meets the selection criterion, a perceptual reference frame is established in the appropriate orientation-perhaps through rotation.... The observer then performs a detailed evaluation of symmetry about the selected axis by explicitly comparing the two halves for mirror-identity.

How the first global, crude, and rapid stage operates is not specified. Nevertheless, this seems to be the essential part of the symmetry-detection mechanism, because the second component process is in fact only consciously controlling what has been signaled preattentively. (For a clear distinction between effortless, immediate, preattentive perception, on the one hand, and attentive search and scrutiny, on the other, see Julesz, 1981.)

\section{Different Zones and Central Presentation}

A second set of factors that have frequently been investigated consists of the contribution of different zones in a pattern to the global impression of symmetry in it, as well as the relative importance of central presentation to it. Most studies have shown that a restricted area around the axis of symmetry is the most important (e.g., Jenkins, 1982) and that central presentation (with the axis of symmetry being located at the point of fixation) is most helpful (e.g., Saarinen, 1988). Nevertheless, several findings urge that this general statement should be qualified.

With respect to the issue of central presentation, Julesz (1971) has noted that the detection of symmetry in simple patterns (e.g., amorphic shapes) does not require that the center of symmetry coincide with the fixation point of the eyes, in contrast with the same detection in complex patterns (e.g., dot textures). Julesz has therefore concluded that symmetry detection operates at two levels: for patterns with low spatial frequencies, the symmetric relations are extracted globally; in contrast, for patterns with high spatial frequencies, the symmetric relations are extracted by a point-by-point comparison process. A similar distinction (without the spatial filtering notions) has been incorporated in most subsequent models and theories (see, e.g., Bruce \& Morgan, 1975; Palmer \& Hemenway, 1978).

With respect to the contribution of different zones, Barlow and Reeves (1979) have provided some evidence for the importance of outer areas also (possibly because of an imaginary contour), and they have stressed the versatility of the symmetry-detection mechanism by showing a remarkable tolerance to smearing (i.e., nonexact correspondences between symmetrically positioned elements) and noncentral presentation. These findings led them to suggest that "symmetry detection requires nothing more than the comparison of dot densities measured over quite large areas symmetrically placed about the putative axis of symmetry" (Barlow \& Reeves, 1979, p. 791). However, they are forced to admit that "each different position of the axis requires a different set of comparisons, and [that] the means by which these sets of comparisons are brought about is not easy to imagine" (p. 792). Again, it seems that this first, preattentive global stage is the real mystery.

\section{Orientational Uniformity and Midpoint Collinearity}

Jenkins's (1983) study of component processes in symmetry detection is a third example of experimental research on the effects of different variables on the detection of symmetry resulting in some basic principles to be taken into account by symmetry-detection models. In fact, he comes closest to the essentials of the first stage of rapid and crude symmetry detection by explicitly manipulating the information that is present in a bilaterally symmetric pattern. Jenkins started from the definition of a bilaterally symmetrical dot pattern "as a twodimensional distribution of uniformly oriented point-pair elements, of nonuniform size, which fall across the same 
axis evenly such that the uniformly oriented pairs have collinear midpoints" (p. 433). In a nice set of experiments, he has shown the visual system's sensitivity to orientational uniformity (i.e., the fact that all virtual lines ${ }^{1}$ connecting the symmetrically positioned dots are parallel) and midpoint collinearity (i.e., the fact that all midpoints of these virtual lines are situated on one straight line, viz., the axis of symmetry). It is tempting to conclude from these findings that symmetry detection is based on these regularities, and Jenkins's own model more or less suggests this.

However, it remains unclear exactly how orientational uniformity and midpoint collinearity are detected. It seems that the detection of both factors must occur cooperatively; in order to detect the orientational uniformity, one has to know how the dots belong together, but to measure the symmetric positions requires information about the orientation of the axis and hence the detection of the midpoint collinearity. Furthermore, experiments with skewed symmetry indicate that orientational uniformity and midpoint collinearity are insufficient to explain symmetry detection.

\section{Skewing}

Skewed symmetry (SS) is the result of a general orthographic projection of BS. In fact, the only situations in which a planar BS in the world is projected to BS on the retina are the accidental cases of an orthogonal viewing position or a plane slanted about an axis perpendicular to the axis of symmetry. In all other cases, BS yields SS in the image plane. In computer vision, the presence of SS in the image is therefore used as a cue to infer BS in the world (see, e.g., Kanade \& Kender, 1983; Stevens, 1979), and algorithms have been proposed to derive constraints on the orientation (slant and tilt) of a nonorthogonal plane from the direction and angle of the skewing (see, e.g., Friedberg, 1986; Hakalahti, 1983). Recently, we have extended this work considerably by allowing interactions between multiple regularities (Van Gool, Wagemans, Vandeneede, \& Oosterlinck, 1990).

As far as we know, the human sensitivity to SS has never been tested formally and systematically (some informal observations were made by Attneave, 1982, and Stevens, 1979). Nevertheless, detecting symmetry in nonorthogonal planes seems to be a prerequisite if a biological visual system is to be able to use this kind of nonaccidental property in object recognition (as proposed recently by Biederman, 1987, and Lowe, 1987). Some preliminary data indicate that, analogously to the aforementioned computer vision algorithms, the human visual system has a perceptual bias toward symmetry that is used in the recovery of a constant three-dimensional (3-D) shape from changing two-dimensional (2-D) images (King, Meyer, Tangney, \& Biederman, 1976).

In a study published elsewhere (Wagemans, Van Gool, \& d'Ydewalle, 1990), we have investigated the perception of SS with a paradigm in which the subjects had to detect and locate an additional dot that disturbed the symmetry (BS or SS) in a dot pattern. As a result of the orthographic projection of $\mathrm{BS}$, the angles between the virtual lines connecting the symmetrically positioned elements and the axis of symmetry generally differ from $90^{\circ}$, but orientational uniformity and midpoint collinearity are preserved by the affine transformation. The results of that study indicate that it is much harder to detect a violation of SS than it is to detect a violation of BS (higher error rates, longer $R T s$ ). In the following section, we will present the principles of a new account of symmetry detection that integrates all the findings reviewed above.

\section{A NEW ACCOUNT AND ITS PREDICTIONS}

Consider the dot pattern presented in Figure 1 (left). Suppose that the human visual system, when confronted with such a pattern, starts processing it by grouping dots together, so that they are eventually represented by virtual lines (see Note 1). Initially, connections will be established in all possible directions, but gradually the local parallelism between the virtual lines spanning the symmetry axis will be noticed. When more and more of these pairwise connections between symmetrically positioned elements are formed, their midpoint collinearity and, in fact, the global symmetry giving rise to it, would be detected (see Figure 1, middle).

\section{Second-Order Relations}

The same pairwise correspondences are possible in SS, yet they are much harder to detect (see Figure 2). The critical difference between both types of patterns occurs in terms of second-order relations holding between pairs of first-order virtual lines. ${ }^{2}$ As can be seen in the right item in Figure 1, two pairs of symmetrically positioned

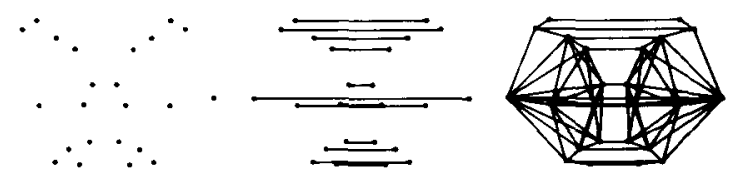

Figure 1. Example of a dot pattern with perfect single symmetry about a vertical axis (left) and its first-order (middle) and secondorder (right) regularities. In the rightmost item, only some of the bootstrapping besed on the correlation quadrangles (i.e., symmetric trapezoids) is shown.

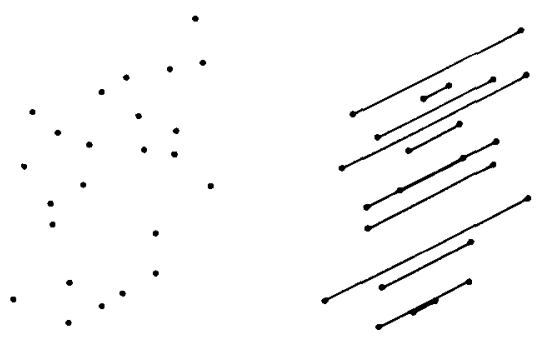

Figure 2. Example of a dot pattern with skewed single symmetry about a vertical axis (left) and its first-order regularities (right). The skewing is $30^{\circ}$ counterclockwise. In contrast with orientational uniformity and midpoint collinearity (Jenkins, 1983), the higher order relations disappear. As a consequence, there are no correlation quadrangles, and hence, no bootstrapping. 
elements constitute a virtual quadrangle with certain special characteristics such as correlated angles. The virtual quadrangles of this basic correlational type are called correlation quadrangles. ${ }^{3}$ In principle, two kinds of such correlation quadrangles are possible: symmetric trapezoids, with two pairs of equal adjacent angles, and parallelograms, with two pairs of equal opposite angles.

Our suggestion is that the normal grouping processes that occur as one of the first stages in all perceptual processing automatically lead to the detection of symmetry, because the possible relations confirm one another, given the Euclidean invariant line lengths (i.e., distances) and angles (i.e., orientations). More specifically, in BS the elements are positioned in such a way that not only the individual elements are in symmetric positions about an axis. The virtual lines formed between two elements not belonging together because of the symmetry, but because of their proximity (i.e., the nonhorizontal lines in Figure 1), have the same lengths and relative orientations as those of their corresponding virtual lines at the other side of the symmetry axis (see Note 3 ).

In SS this additional support from connections between nonsymmetrically positioned elements is absent, because all lengths and angles of virtual lines representing them are different as a result of the affine skewing transformation. Although all first-order relations (defined in terms of the "correct" correspondences) are the same for BS and SS, the second-order relations (dependent on the "incorrect" correspondences as well) are regular only for BS. So, given that the visual system does not "know" initially which pairwise connections indicate symmetric correspondences, many spurious ones are made. These are helpful in BS, but not in SS.

These geometric relations holding in patterns with BS might facilitate the detection of it in the following way. Let us assume that from the moment at which some parallelism is detected (i.e., first-order regularity), a local reference frame is established for it (one axis of the frame parallel to it, the other orthogonal to it), with respect to which all other angles are expressed (i.e., second-order regularity). Note that this does not require that the axis of symmetry be detected for the second-order regularities to be registered. If the quadrangle formed in this manner is of a nonaccidental nature (i.e., the genuine result of the regularity in the pattern), it can serve as a starting point for building additional quadrangles. The propagation of this local reference frame, called bootstrapping, is quite easy for symmetric trapezoids, because one of the two axes of the frame indicates the direction in which to proceed.

Without becoming too engaged with the specific details of processing, we can say that all previous findings regarding effects of multiple axes, different zones in a pattern, and central presentation can be accounted for if we assume that the normal grouping processes start in parallel, but operate faster in the center (probably because of higher foveal accuracy, although additional reasons might exist; cf. Saarinen, 1988). Similarly, the orientational aniso- tropy found in symmetry detection can be incorporated as a weighted parameter expressing the ease of the spreading of the grouping in different directions. In addition to this post hoc explanatory power, our account leads to quite specific predictions that have not been tested so far. In the experiment reported here, we investigated the importance of the higher order relations by disturbing them and measuring the detectability of the symmetry in the resulting patterns. The way to do this is to use symmetric patterns with multiple axes and skewing.

\section{Multiple Symmetry}

In single symmetry, a pattern is reflected about a single axis. In the present study, in which all patterns have 24 dots, the original input pattern is a pseudorandom collection of 12 dots, and the axis is oriented vertically, horizontally, or diagonally to the left (L) or to the right (R). When the reflection occurs orthogonally to the axis, the resulting symmetry is a perfect BS. When the reflection is performed in a nonorthogonal coordinate system, the resulting symmetry is skewed single symmetry (for a more mathematical treatment of skewing, see Friedberg, 1986). In multiple symmetry, a pattern is reflected about more than one axis. For example, in our double symmetry, represented in Figure 3, a pseudorandom collection of six dots is reflected about a V-H system of axes or an L-R system of axes. In our quadruple symmetry, shown in Figure 5, a pseudorandom collection of only three dots is reflected about each of the four possible axes $(\mathrm{V}, \mathrm{H}, \mathrm{L}$, and $\mathrm{R})$. Of course, the skewing transformation can be applied equally well to these multiple symmetries. The results are called skewed double symmetry (Figure 4) and skewed quadruple symmetry (Figure 6), respectively.

\section{Predictions}

It is interesting to see what happens with the higher order relations in all these different kinds of symmetry with single or multiple axes and with skewing. The number of virtual quadrangles along each axis present in a pattern is constant. This number is $[N(N-1)] / 2$; with $N$ being 12 (i.e., the number of dot pairs), this yields 66 virtual quadrangles. ${ }^{4}$ The different kinds of symmetries differ in the number of axes along which there are 66 quadrangles and in the regularity of the quadrangles. In Table 1, the available quadrangles are specified for all of the kinds of symmetries studied in the experiment reported here.

In perfect double symmetry, two axes exist, each with 66 virtual quadrangles, all of which are of the basic correlational type. As can be seen in Figure 3, there are 66 correlation quadrangles, 60 symmetric trapezoids, and 6 even higher order ones (viz., rectangles with four correlated angles; see Notes 2 and 3 ), all $2 \times 2$ identical. ${ }^{5}$ In skewed double symmetry, however, only 6 parallelograms (viz., skewed rectangles) and 60 nonsymmetric trapezoids are present along each of the two axes (see Figure 4). So the number of quadrangles of the correlational type is significantly decreased by skewing. The 

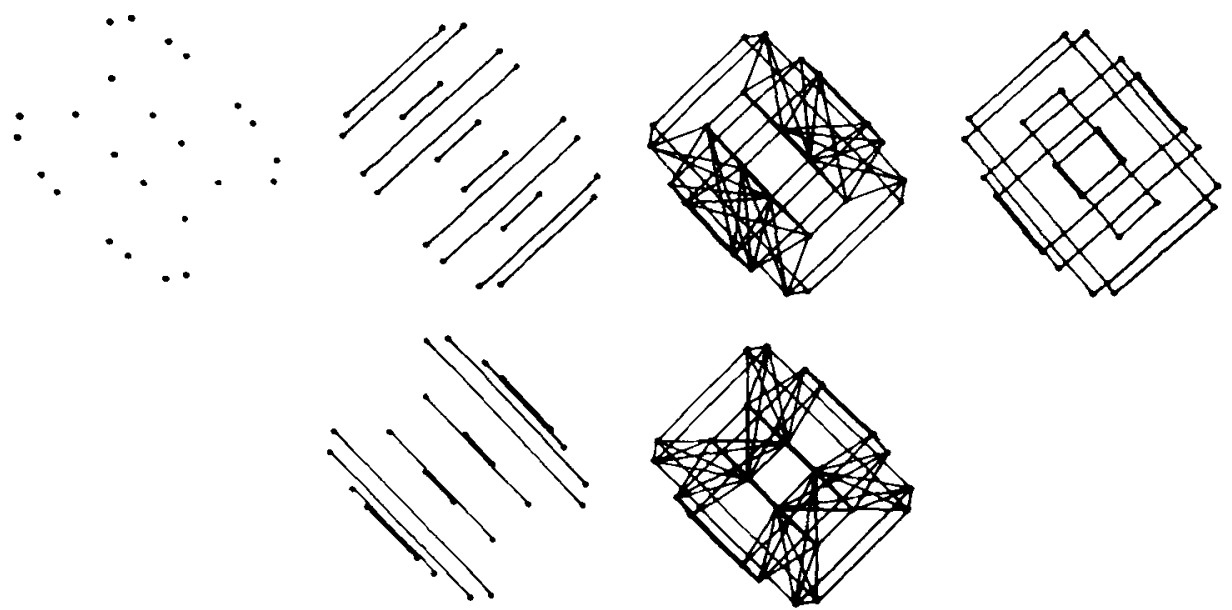

Figure 3. Example of a dot pattern with perfect double symmetry about left and right axes. In the second column, the first-order regularities are shown for both axes separately. In the third column, the bootstrapping based on the second-order regularities is shown for the same two axes. The top-right item represents the correlation quadrangles that result from combining the two first-order regularities (i.e., rectangles).
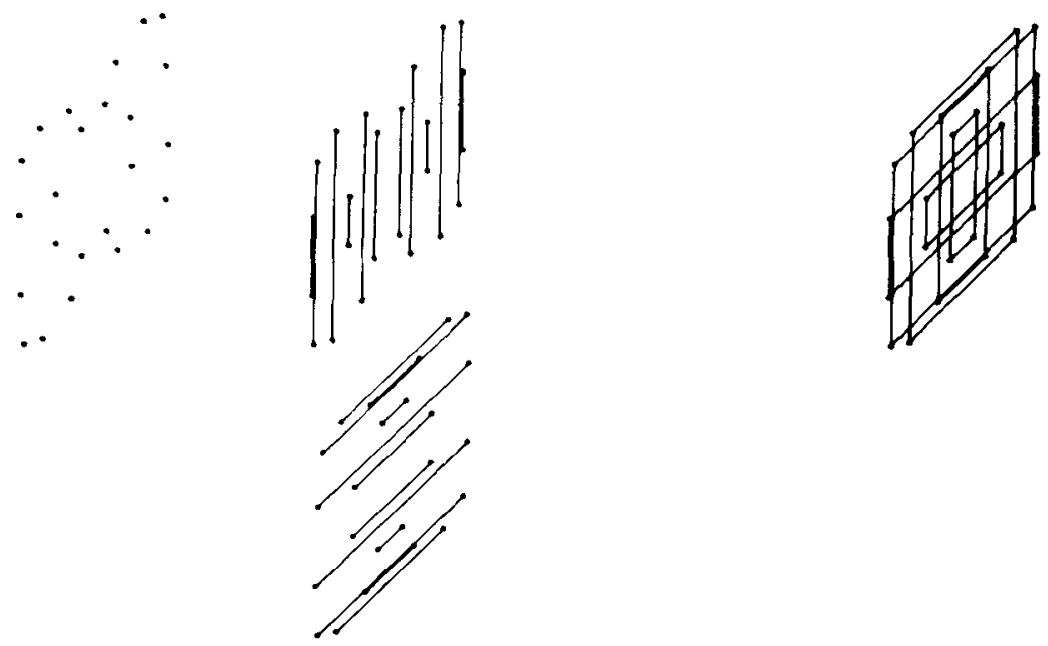

Figure 4. Example of a dot pattern with skewed double symmetry about vertical and right axes, as a result of a $45^{\circ}$ counterclockwise skewing of a double vertical-horizontal symmetry about the vertical axis. In the second column, the first-order regularities are shown for both axes separately. Their superposition gives the parallelograms shown in the rightmost item, but there is no bootstrapping between them.

prediction, then, is that there will be a significant difference in detectability between the perfect and the skewed double symmetries.

In perfect quadruple symmetry, four axes are present, each with 66 virtual quadrangles, all of which are again of the basic correlational type. As one can see in Figure 5, there are 60 symmetric trapezoids and 6 rectangles along each axis, all $2 \times 2$ identical. In skewed quadruple symmetry, there are also four axes with 66 virtual quadrangles, but not all of them are correlation quadrangles (see Figure 6). Along two of the four axes, there are 60 sym- metric trapezoids and 6 rectangles, all $2 \times 2$ identical. Along the other two axes (i.e., the ones around which the skewing is applied), there are 60 nonsymmetric trapezoids and 6 parallelograms, again pairwise identical. In fact, skewing a quadruple symmetry results in a perfect double symmetry (the orientation of the orthogonal symmetry axes depends on skewing angle and axis) and a skewed double symmetry. All in all, the number of correlation quadrangles is very high, so that the detection of this kind of symmetry must be very easy, at least if the account presented above has some validity. 

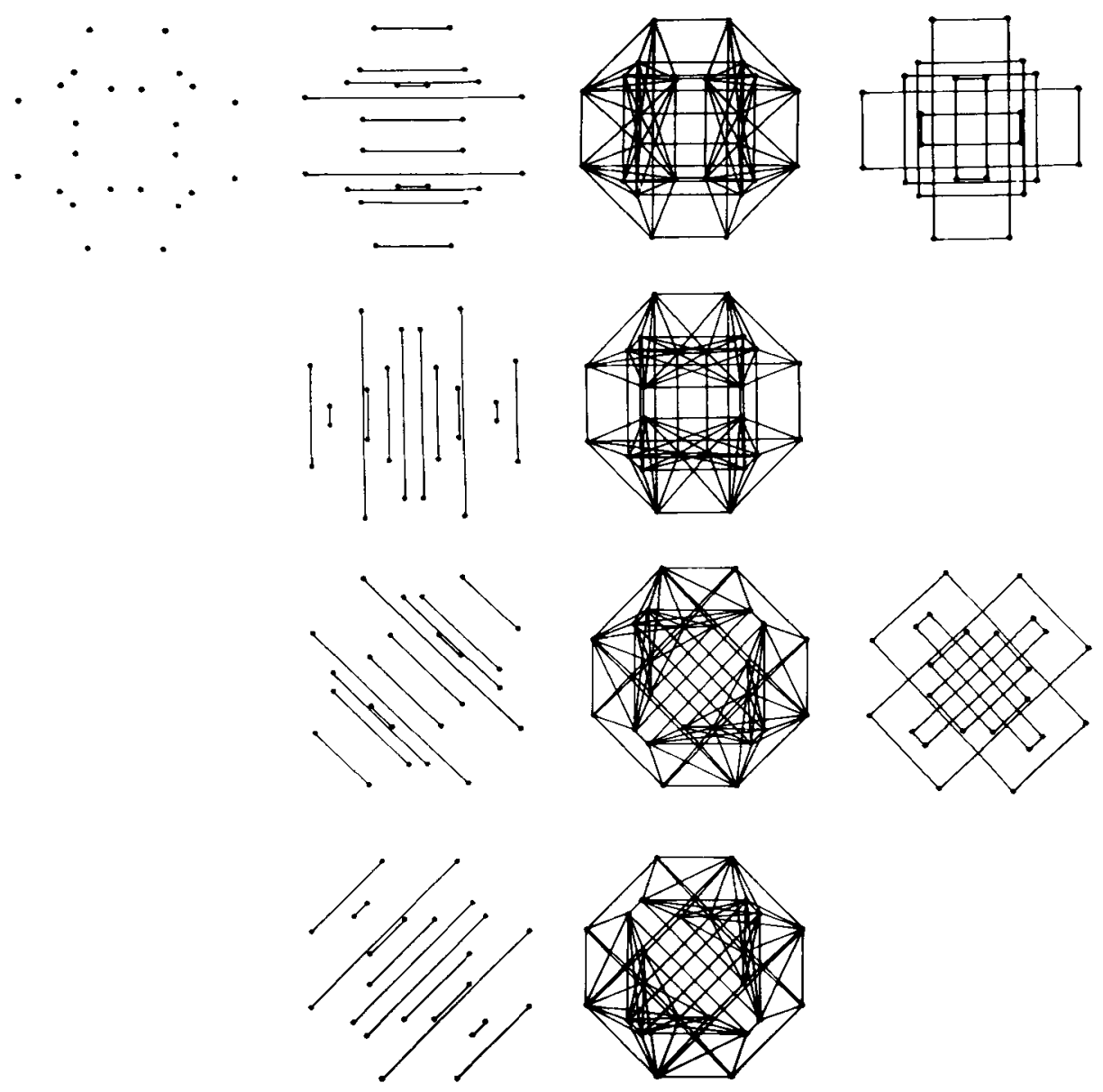

Figure 5. Example of a dot pattern with perfect quadruple symmetry (i.e., about vertical, horizontal, left, and right axes). In the second and third columns, the first-order regularities and the bootstrapping based on the second-order regularities are shown, respectively, for all four axes. The fourth column represents the rectangles resulting from combining two orthogonal first-order regularities.

To recapitulate what is hinted at above, in perfect single symmetry 66 correlation quadrangles are present along the axis; but they are not pairwise identical, and there are no rectangles, only symmetric trapezoids (see Figure 1). In skewed single symmetry, the second-order relations are destroyed, and hence, no correlation quadrangles are present whatsoever (see Figure 2). In summary, the skewing should have little or no effect on the detection of symmetry in dot patterns with four axes, a small but maybe significant influence on the detectability of double symmetry, and a large disruptive effect when only a single axis is present.

Our previous study (Wagemans et al., 1990) showed that skewed single symmetry is very hard to detect. In fact, preattentive detection of regularity was completely destroyed by skewing (these dot patterns look random at first sight). Subsequently, we have used SS as a means of investigating orientational effects and component processes in symmetry detection without the confounding of two orientations (axis and virtual lines) and the cooperation of two stages (global axis selection and local point-by-point evaluation) that are clearly present in BS (Wagemans, Van Gool, \& d'Ydewalle, in press).

However, a fundamental weakness of those studies with respect to the normal process of preattentive symmetry detection was that the experimental paradigm forced the subjects to search for a particular regularity (or a dot disturbing it) consciously, yielding very long search times (even up to 20 or $30 \mathrm{sec}$ ). To investigate the role of these higher order relations more quantitatively in a paradigm measuring fast symmetry detection was our principal aim in the experiment reported here. Following Julesz's (1981) operational definition, the requirement of the level of the process being measured (preattentive vs. attentive) has been met by presenting the dot patterns tachistoscopically $(100 \mathrm{msec})$ in a discrimination task (random vs. symmetric). The requirement of quantitative detail has been met by using different kinds of symmetries. As indicated above, it appears that the identity and number of higher order regularities can be varied sufficiently by skewing single, double, and quadruple symmetries. 

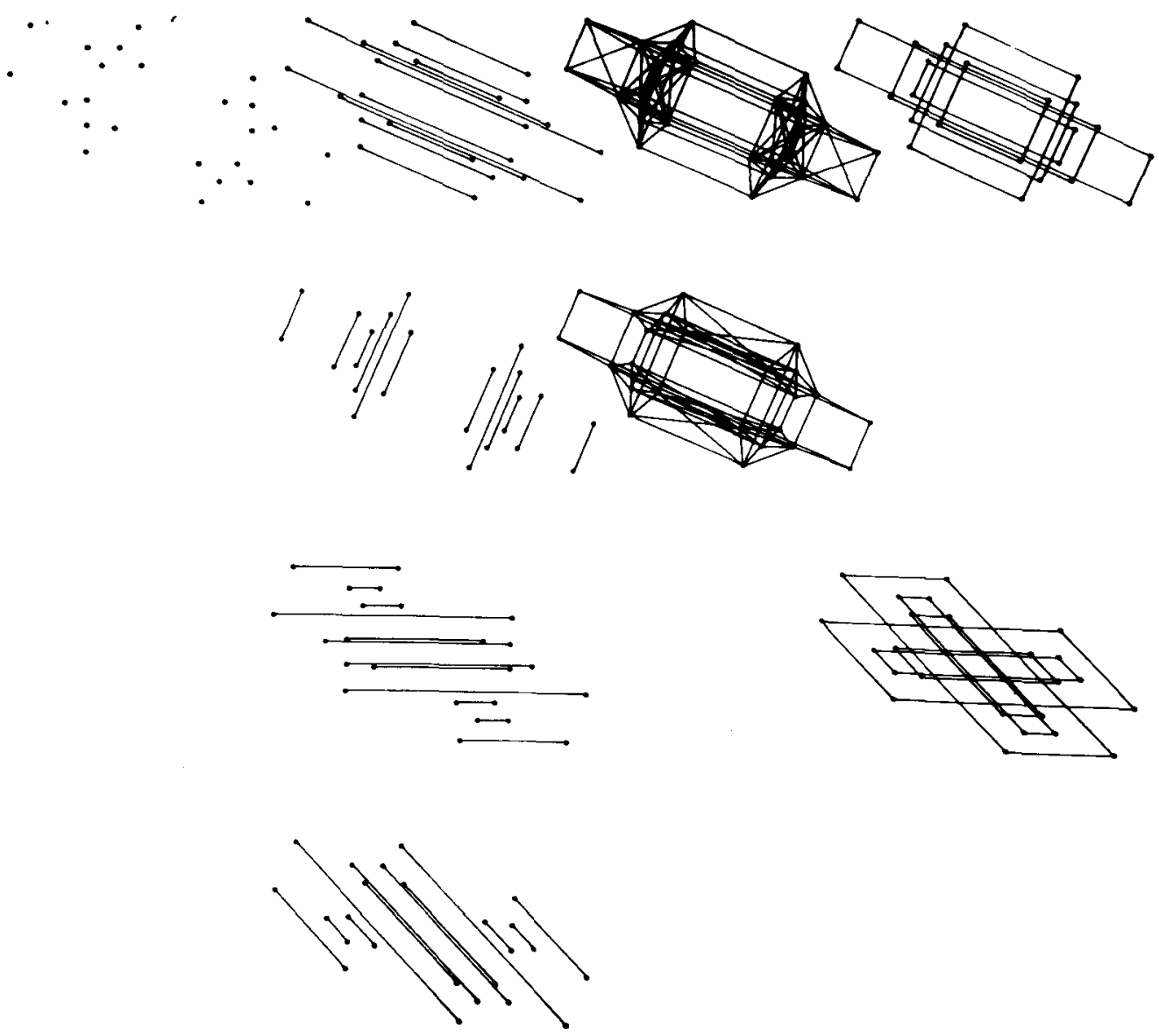

Figure 6. Example of a dot pattern with quadruple symmetry after $45^{\circ}$ clockwise skewing about the left axis. As a result, there is skewed symmetry about the left and horizontal axes and perfect double symmetry about intermediate axes. In the second column, the first-order regularities for the four axes are shown, whereas the third column indicates that there is only bootstrapping based on the second-order regularities for the two axes with perfect symmetry. In addition, superposition yields rectangles for the perfect double symmetry and parallelograms for the skewed double symmetry.

\section{Subjects}

Four subjects participated in the experiment: the first author and 3 naive observers who were paid about \$4 US per hour. All observers had normal or corrected-to-normal vision. Because of the very large number of trials and conditions (see the Procedure section), only a few well-motivated subjects (subjects who were willing to return several times) could be run. The expectation was that the processes under investigation are so basic as to allow no cognitive biases or large interindividual differences. Previous research has indicated that despite overall differences between trained and untrained subjects, the same relative detectabilities of different kinds of symmetries are usually obtained (Locher \& Wagemans, 1991; Royer, 1981; Wagemans et al., in press).

\section{Task}

The subjects received a large number of random and symmetric dot patterns, randomly intermixed, which had to be judged as regular or not (the notion of "symmetry" was avoided in the instructions to the subjects, because naive observers tend to equate this with perfect bilateral symmetry only). The yes/no answers had to be made as accurately as possible. There was no time pressure, except that the dot patterns were presented only for a fixed small amount of time (i.e., $100 \mathrm{msec}$ ).

\section{Stimuli}

The random dots used to generate the symmetric dot pattems were only partly random. There were three basic constraints. First, the random dots had to fall within a global circular area defined around the center of the screen with a diameter of $10 \mathrm{~cm}$, and they had to be located so that their symmetrically positioned partners also fell within the same circular area. Second, the random dots had to be located outside a circular area defined locally around the other dots in the pattern (both the random and the symmetric ones) with a diameter of $1 \mathrm{~cm}$. The third constraint on the locations of the random dots was that they had to be distributed equally within the total area. This was realized through the location of an equal number of dots (i.e., eight) within each of the three annuli that resulted from four concentric circles defined around the middle of the screen. The radii of two of the four circles were already fixed by the first two constraints; the outermost circle had a radius of $5 \mathrm{~cm}$, and the innermost circle had a radius of $0.5 \mathrm{~cm}$. The remainder of the whole circular area was divided into three annuli with almost equal areas by two circles with radii of 2.75 and $4.25 \mathrm{~cm}$. 
Table 1

Enumeration of Available Virtual Quadrangles in All Kinds

of Symmetry Generated by Combining Different Numbers and Orientation of Axes with Different Skewing Angles

\begin{tabular}{lcccc}
\hline & $\begin{array}{c}\text { Symmetric } \\
\text { Trapezoids }\end{array}$ & Rectangles & $\begin{array}{c}\text { Nonsymmetric } \\
\text { Trapezoids }\end{array}$ & Parallelograms \\
\hline $\begin{array}{l}\text { Perfect quadruple } \\
\text { Skewed quadruple }\end{array}$ & $4 \cdot \frac{60}{60}$ & $4 \cdot \underline{6}$ & $2 \cdot 60$ & $2 \cdot 6$ \\
$\begin{array}{l}\text { Perfect double } \\
\text { Skewed double }\end{array}$ & $2 \cdot \frac{6}{60}$ & $2 \cdot \frac{6}{6}$ & $2 \cdot 60$ & $2 \cdot 6$ \\
$\begin{array}{l}\text { Perfect single } \\
\text { Skewed single }\end{array}$ & 66 & & 66 & \\
\hline
\end{tabular}

Note-Underlined numbers indicate that they are pairwise identical. As with all $n$ th-order regularities with $n>2$, we do not know whether they are used by our visual system, but they are available information.

Apart from the first constraint, which defined the visual angle of the stimulus field, the constraints on the random selection of dot locations were introduced to prevent special grouping effects. Without the second constraint, some dot clusters might have arisen that would have been processed as a single higher order feature. Although this feature clustering is in itself an interesting topic for study (see, e.g., Jenkins, 1983; Locher \& Wagemans, 1991; Wagemans et al., 1990), it would have been a confounding factor in this experiment. Without the third constraint, many patterns would have been generated with an almost completely empty inner zone and most dots constituting an imaginary outer contour; the restriction on the interdot distances forces the dot-selection process to locate the dots in the periphery of the circular area, because this maximizes the chance that a dot does not fall within the local area around another dot. Again, this imaginary contour is in itself an interesting topic for study (see, e.g., Barlow \& Reeves, 1979; Wagemans et al., 1990), but it was not the focus of the present experiment. In our experiment, the special grouping effects could have functioned as cues to distinguish symmetric from random dot patterns and were, therefore, avoided.

In summary, the symmetric dot patterns were generated accordding to the following procedure. First, a random dot was selected within the first annulus (defined as the region between the circle with radius $0.5 \mathrm{~cm}$ and the circle with radius $2.75 \mathrm{~cm}$ ). This random dot was projected according to the kind of symmetry one wanted to generate (e.g., reflected seven times for a quadruple symmetry). Next, it was checked to see whether the projected dot(s) was (were) not too close to the original dot (or the other projected dots). Then, a second random dot was selected, this time in the second annulus (defined as the region between the circle with radius $2.75 \mathrm{~cm}$ and the circle with radius $4.25 \mathrm{~cm}$ ). Again, the location of this dot was checked to ensure that it was outside the local area around an already selected dot. Then again, this random dot was projected according to the appropriate symmetry rule and checked. A new dot was selected randomly, this time in the third annulus, and so on until 24 dots were located pseudorandomly and symmetrically in the three annuli in the circular area.

When an SS had to be generated, a second phase was added to this first phase of random dot selection and symmetric projection. In accordance with one of six possible skewing rules to be followed, a clockwise (CW) or counterclockwise (CCW) skewing of a particular angle $\left(15^{\circ}, 30^{\circ}\right.$, or $\left.45^{\circ}\right)$ was performed about a particular axis (V, H, L, and R). After this skewing, a final check was made on the minimal interdot distances.

The general procedure for stimulus generation of the random dot patterns was as similar to the one for the symmetric patterns as it could be. The constraints on the randomization of the dot locations were the same. Now, 24 random dots were to be located in the circular area with a diameter of $10 \mathrm{~cm}(8$ dots in each annulus). After every random generation of a possible dot location, a check on the minimal interdot distances was made. The skewing transformation was also applied to the random dot patterns, because the circular area in which the dots were located (and to which the subject's attention had to be divided) was transformed to an elliptic one in the case of SS. Its elongation measure and direction were dependent on the angle and direction of the skewing and on the orientation of the axis about which the skewing was applied. Work by Lansky, Yakimoff, and their colleagues has shown that human observers are quite good at estimating the orientation of an elliptic dot pattern (e.g., Lansky, Yakimoff, \& Radil, 1987; Lansky, Yakimoff, Radil, \& Mitrani, 1989). Therefore, in order for the form of the stimulus zone not to be a potential cue for the decision on the randomness or regularity of the pattern constituted by the dots located in the zone, the random patterns had the same stimulus fields as did their symmetric counterparts.

\section{Apparatus}

The dot patterns were generated by a C program on a SUN-3 Workstation with a Motorola MC 68881 floating-point board. Stimulus presentation was automated by another $C$ program on an IBM-AT-compatible with a Phoenix 80386 processor and a VISTA card. The stimuli were presented on a raster display with high temporal and spatial resolution (BARCO, Type CDCT-6351B) used in PAL mode with a $50-\mathrm{Hz}$ temporal resolution and a $740 \times 578$ spatial resolution, noninterlaced, as black dots against a homogeneous gray background. The experimental room was completely darkened, and screen borders were covered by black cardboard with a circular aperture to reduce orientational cues. The subjects were seated at a distance of $114 \mathrm{~cm}$ with their eyes in front of the middle of the screen. At that distance, the size of the individual dots and the whole patterns subtended $5.7^{\prime}$ and $5^{\circ}$, respectively. Forehead- and chinrests were used to prevent head rotations.

\section{Procedure}

The experiment was designed as a signal-detection experiment. The "signal" to be detected was symmetry or, as it was explained to the subjects, regularity. Before starting the experiment, the subjects received a rather extensive introduction to what was meant by "regularity." Examples of each of the different symmetries were shown (on paper), and time was given to explore the dot patterns sufficiently to detect the presence of single or multiple axes. They were then instructed that they would see many such patterns, mixed with random dot patterns and presented only very briefly. The task was described as a forced choice in response to the question, "Is it a regular pattern or not?" - which had to be answered to the best of their abilities. Response fingers were chosen by the subjects. All 4 subjects answered "yes" (regular) with the right index finger and "no" (random) with the left index finger.

A trial consisted of the following sequence of events. First, a fixation pattern was presented for $500 \mathrm{msec}$. This was a black " +" 
at the center of the imaginary circle on a homogeneous gray background. It allowed the subject to fixate the center of the dot pattern when it was presented immediately thereafter. Second, a dot pattern was presented for $100 \mathrm{msec}$. This was a collection of 24 random or symmetric black dots on a homogeneous gray background falling within an imaginary circle or ellipse centered at the middle of the screen (see Stimuli section above). Immediately following this 100 -msec dot pattern, a masking pattern was presented for 1,500 msec. This was a similar black-on-gray collection of 36 random dots sufficiently large to cover the stimulus pattern completely. A set of 10 masking patterns was used. After each stimulus pattern, one of these 10 masks was randomly selected. The reason why the same mask was not used after all stimuli is that it would thereby have lost its masking power. From the moment the mask was on, the subject could respond by pressing one of the two buttons on a response panel connected to the PC configuration used for the stimulus presentation. Each answer was instantaneously evaluated by the computer so that immediate feedback could be given. A correct answer was followed by a $300-\mathrm{msec}$ high-frequency tone $(750 \mathrm{~Hz})$, a false response by a 500 -msec low-frequency tone $(100 \mathrm{~Hz})$. The only reason why this feedback was provided was to keep the motivation and arousal of the subjects at an optimal level. As a result of this feedback, some learning probably occurred, especially for the naive observers. This potential secondary effect was controlled for by randomizing trial and block orders across subjects.

Following the introductory session in which the concept of regularity was explained and demonstrated and in which the task was described, subjects received a practice session of three series of 158 trials. A practice series contained one symmetric and one random dot pattern for each of the 79 kinds of symmetry. This odd number is the result of combining three variables as orthogonally as possible: (1) the number of axes (three levels, viz., one, two, and four); (2) the orientation of the axis (four levels when there was one axis, viz., V, H, L, and R; two levels when there were two axes, viz., V-H and L-R; and only one level when there were four axes); and (3) the skewing angle (seven levels, viz., $15^{\circ}, 30^{\circ}$, and $45^{\circ}$, both $\mathrm{CW}$ and $\mathrm{CCW}$, in addition to $0^{\circ}$ skewing, i.e., yielding perfect symmetry).

Although this nonorthogonal combination complicated the data analysis somewhat, we considered this to be a smaller disadvantage than the alternative solution, which would have been to present four blocks of perfect quadruple symmetry and two blocks of each type of double symmetry. Because the prediction was that these were the easier conditions, presenting them more than once would have left room for a more mundane explanation for that finding, in that these types of symmetry could have been learned better than the other conditions.

The data for the practice stimuli were not analyzed. The only reason for the practice session was to allow the subjects to get an impression of the difficulty of the task (i.e., the short presentation time) and to exercise the procedure (e.g., the sequence of events that defined a trial, the feedback, etc.). The same patterns were not used in the experimental sessions.

Trials were presented in blocks of 210: Ten practice trials (different from the experimental ones and not analyzed) preceded 100 random and 100 symmetric experimental ones. The kind of symmetry was constant within and changing between blocks. The order of trials within a block and the order of blocks were randomized for each subject separately. Following each block of 200 trials, the subject was informed about performance level (percent correct) for that block, again to maintain motivation.

After each block, the subject could choose to continue or to quit. They were aware of the large number of trials to be run (i.e., 17,064; viz., 79 blocks of 200 experimental trials, together with the $3 \times$ 158 trials from the practice sessions and the $79 \times 10$ practice trials preceding the experimental blocks). This encouraged them to do more than a few blocks each time they were in the lab. The sub- jects were advised to take short breaks between the blocks. On the average, subjects did 5-10 sessions of 7-15 blocks, spread over a period of 4-7 days. The duration of the whole experiment (i.e., the introductory session, practice session, and 79 experimental blocks) was about $13 \mathrm{~h}$ for each subject.

\section{RESULTS}

For each observer and condition, a fourfold detection table was constructed from the responses. On the basis of these raw data, several signal-detection measures such as $d^{\prime}$ were calculated. On the set of $d^{\prime}$ values for all conditions and for the 4 subjects, an ANOVA was performed to assess the differences in detectability between the different kinds of symmetry. ANOVAs on other measures, such as raw hit and false-alarm rates, yielded similar conclusions and will not be reported here.

The data points for the perfect multiple symmetries were repeated for each axis orientation (i.e., perfect quadruple symmetry was repeated four times, and both perfect double symmetries twice each), so that the data could be analyzed for the different conditions as an orthogonal combination of three factors: number of axes (three levels: four, two, and one), axis orientation (four levels: V, H, $\mathrm{L}$, and $\mathrm{R}$ ), and skewing angle (seven levels: $-45^{\circ},-30^{\circ}$, $-15^{\circ}, 0^{\circ}, 15^{\circ}, 30^{\circ}$, and $45^{\circ}$ ). This "trick" has consequences for the variability in the corresponding cells only when the effects of axis orientation are analyzed. This causes no serious problems, because orientation was of secondary importance in this study. Moreover, several alternative tests (e.g., in nested designs) with correct numbers of observations at each level have been performed and have yielded very similar results.

In addition to this overall analysis, a posteriori comparisons (Tukey's $H S D$ tests) were done to evaluate all pairwise differences for the main effects and at all levels of each factor interacting with other factors. The results for these follow-up tests will be summarized only descriptively (all quantitative details can be provided on request). Furthermore, a restricted number of a priori comparisons ( $F$ and $t$ tests) were performed to test the specific predictions of the model formulated above. Throughout, the level of statistical significance was set at $p<.05$, unless reported otherwise.

\section{Overview}

All main effects and interactions are (at least marginally) statistically significant. The average $d^{\prime}$ values for the different kinds of symmetry and the results of the ANOVA associated with them can be found in Tables 2 and 3, respectively. As can be seen, the detectability of the symmetry decreases as the number of axes decreases, as the orientation of the axis changes from $V$ and $H$ to $L$ and $R$, and as the skewing angle increases. However, because of the significant interactions between these factors, one must be careful about interpreting these effects. It appears, for example, that axis orientation and skewing angle do not have an effect on the detectability of symmetry when there are four axes of symmetry. 
Table 2

\begin{tabular}{|c|c|c|c|c|c|c|c|c|}
\hline & \multicolumn{8}{|c|}{ Skewing Angle } \\
\hline & $-45^{\circ}$ & $-30^{\circ}$ & $-15^{\circ}$ & $0^{\circ}$ & $15^{\circ}$ & $30^{\circ}$ & $45^{\circ}$ & $M$ \\
\hline \multicolumn{9}{|l|}{ Number } \\
\hline 4 & 4.57 & 4.55 & 4.65 & 5.09 & 4.76 & 4.52 & 4.54 & 4.67 \\
\hline 2 & 2.30 & 3.20 & 3.90 & 4.60 & 3.99 & 3.19 & 2.36 & 3.36 \\
\hline 1 & 0.51 & 1.06 & 2.48 & 3.40 & 2.64 & 0.99 & 0.82 & 1.70 \\
\hline \multicolumn{9}{|l|}{ Orientation } \\
\hline Vertical & 2.52 & 3.42 & 3.85 & 4.81 & 4.09 & 2.85 & 2.70 & 3.46 \\
\hline Horizontal & 2.63 & 3.00 & 3.61 & 4.51 & 3.83 & 3.23 & 2.67 & 3.35 \\
\hline Left & 2.22 & 2.40 & 3.59 & 4.23 & 3.63 & 2.62 & 2.67 & 3.05 \\
\hline Right & 2.47 & 2.92 & 3.65 & 3.91 & 3.65 & 2.91 & 2.24 & 3.11 \\
\hline$M$ & 2.46 & 2.93 & 3.67 & 4.37 & 3.80 & 2.90 & 2.57 & \\
\hline
\end{tabular}

Table 3

Results of Analysis of Variance on Detectability Scores $\left(d^{\prime}\right)$

\begin{tabular}{lrrrr}
\hline \multicolumn{1}{c}{ Effect or Interaction } & \multicolumn{1}{c}{$d f$} & \multicolumn{1}{c}{$F$} & \multicolumn{1}{c}{$p$} \\
\hline Number of axes & 2,6 & 103.55 & 0.00017 \\
Axis orientation & 3,9 & 3.72 & 0.054 \\
Skewing angle & 6,18 & 37.93 & 0.00000 \\
Number of axes $\times$ axis orientation & 6,18 & 2.68 & 0.049 \\
Number of axes $\times$ skewing angle & 12,36 & 13.66 & 0.00000 \\
Axis orientation $\times$ skewing angle & 18,54 & 1.81 & 0.048 \\
Number of axes $\times$ axis orientation $\times$ skewing angle & 35,108 & 1.45 & 0.073 \\
\hline
\end{tabular}

To be able to judge what happens exactly with the higher order interactions, the effects of skewing angle and axis orientation on the detectability of the symmetry are shown for each number of axes in Figure 7 (the top, middle, and bottom rows represent the results for quadruple, double, and single symmetry, respectively). In addition to the results across all subjects (left column), the data for two individuals (the first author and a naive subject) are shown to give an idea of the interindividual variance (middle and right columns, respectively). It appears that the basic trends were very similar for the separate subjects.

\section{Main Effects}

Number of axes. Across all axis orientations and skewing angles, symmetry is easier to detect with an increasing number of axes. A posteriori comparisons showed all pairwise differences to be significant: Quadruple symmetry was easier than double symmetry, which was easier than single symmetry.

Axis orientation. Although the main effect of axis orientation was only marginally significant $(p<.06)$, it is interesting that the combined detectability for the two main axes ( $\mathrm{V}$ and $\mathrm{H}, d^{\prime}=3.41$ ) was somewhat higher than the one for the two diagonal axes [L and $R, d^{\prime}=$ $3.08 ; F(1,3)=7.75, p<.07]$.

Skewing angle. Most pairwise differences for this factor were significant, at least all those between two nonsubsequent levels (e.g., between $0^{\circ}$ and $30^{\circ}, \mathrm{CW}$ or $\mathrm{CCW}$ ). An alternative way to assess the effect of skewing angle is to use trend analysis. The quadratic trend was the most reliable one, but there was a small quadratic component added to it $[F(1,3)=239.94$ and 11.00 , respectively]. In this case, this means that symmetry becomes more difficult to detect, with increasing skewing angle, both $\mathrm{CW}$ and $\mathrm{CCW}$. The quadratic component was caused by the fact that the decrease in detectability from $30^{\circ}$ to $45^{\circ}$ skewing angles was less than expected on the basis of linearity (probably because of orientational effects of the virtual lines resulting from combining axis orientation with skewing angle; see Wagemans et al., in press).

\section{Interactions}

Number of axes $\times$ axis orientation. This interaction means that, averaged across perfect and skewed symmetries, the orientation of the axis did not matter for quadruple symmetry $[F(1,3)<1]$, whereas a clear orientational effect was found for double symmetry $[F(1,3)=$ 69.99 , with the main axes $\left(d^{\prime}=3.64\right)$ yielding higher detectability than the oblique axes $\left(d^{\prime}=3.08\right)$. For single symmetry, the orientational rank ordering was similar (single $\mathrm{V}$ and single $\mathrm{H}, d^{\prime}=1.95$, vs. single $\mathrm{L}$ and single $\mathrm{R}, d^{\prime}=1.45$ ), but it does not approach statistical significance $[F(1,3)=2.23, p>.20]$.

Number of axes $\times$ skewing angle. As can be inferred from Table 2, this interaction means that, averaged across axis orientations, the skewing angle did not matter for quadruple symmetry $(p>.15)$, whereas the effect of skewing on the detectability of symmetry became increasingly more dramatic for the double and the single symmetries $[F(1,3)=3.27,19.66$, and 61.48 , respectively $]$. 

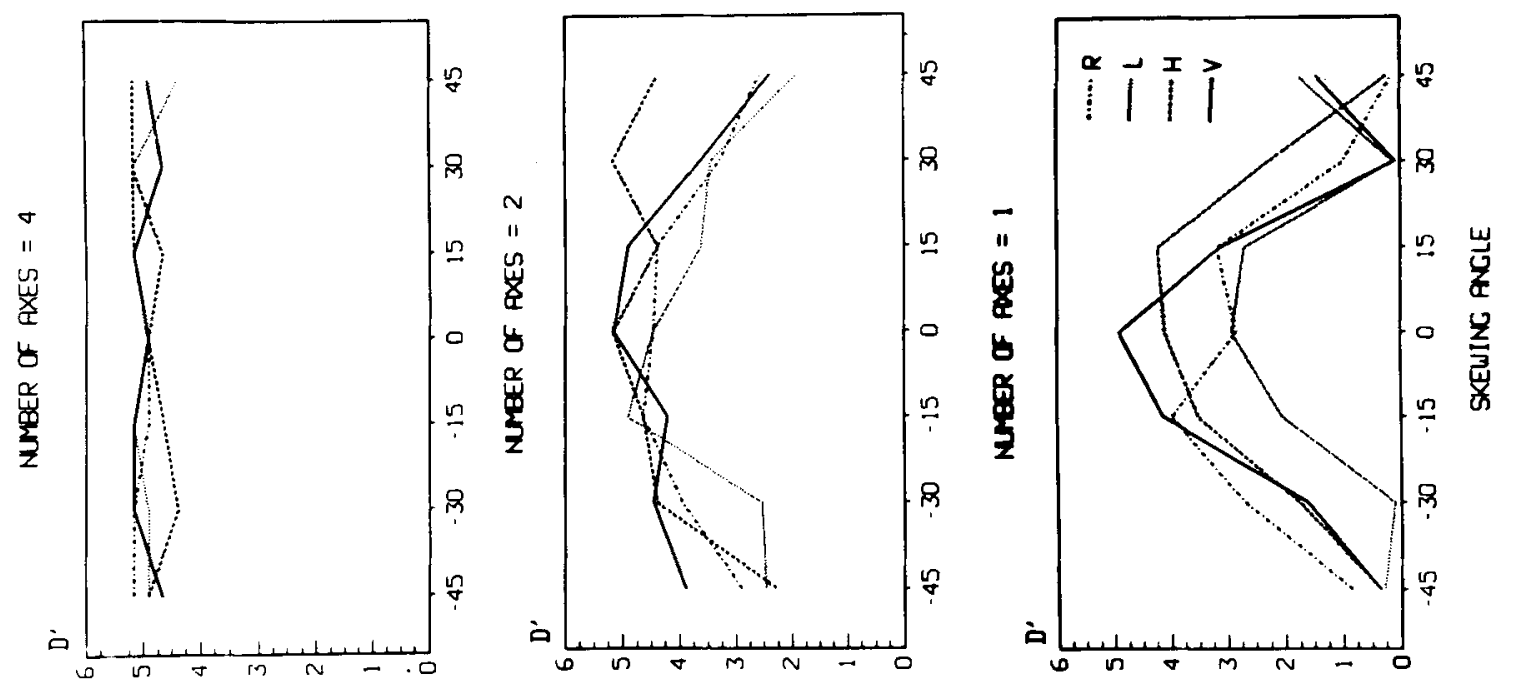

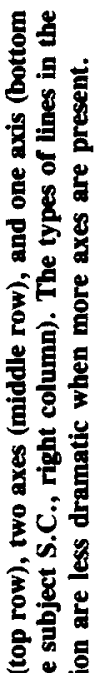
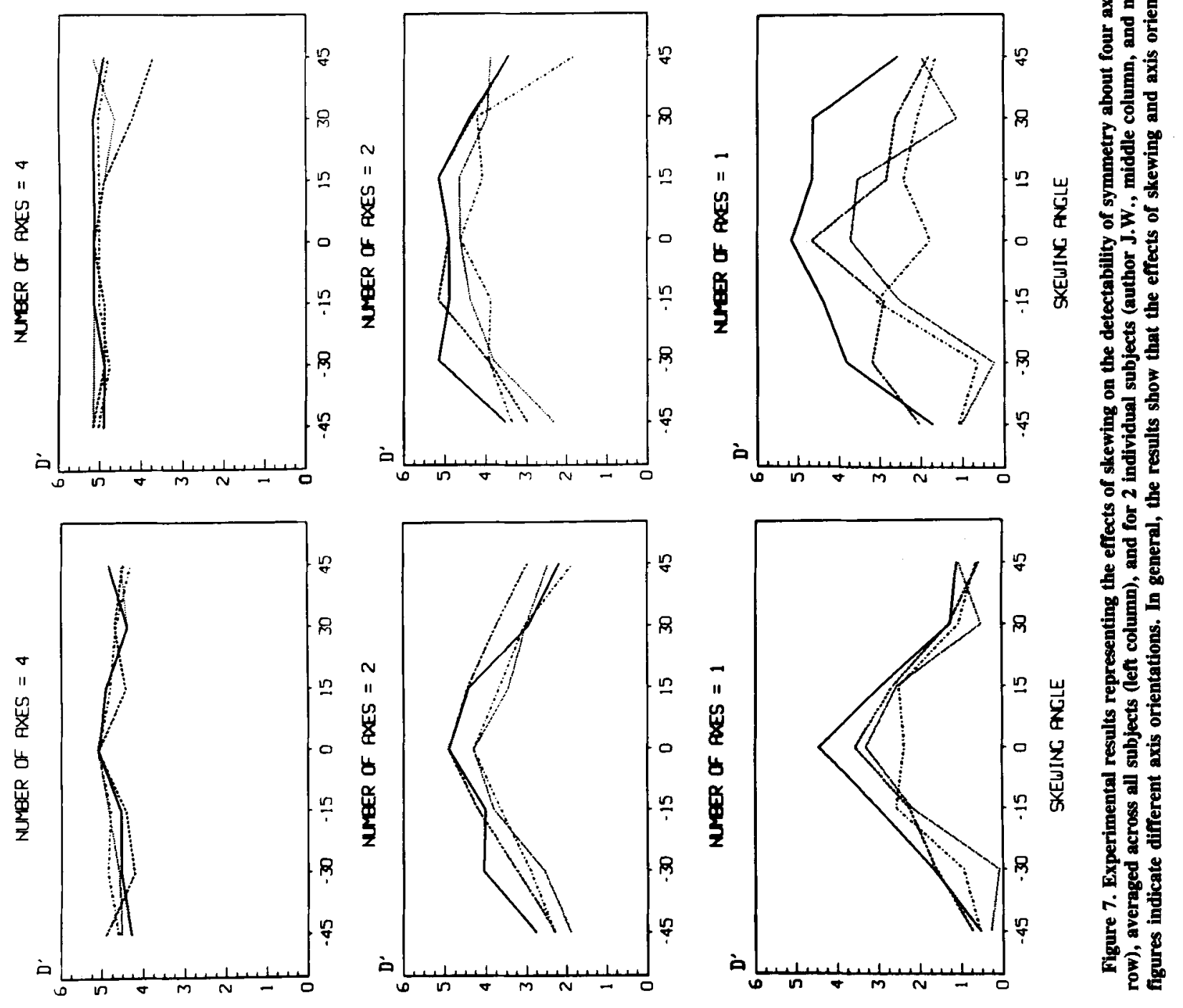
When only one axis of symmetry was present in a pattern, skewing about it by a large angle (i.e., $30^{\circ}$ or $45^{\circ}$ ) reduced the detectability considerably $\left(d^{\prime}=0.84\right)$.

Axis orientation $\times$ skewing angle. The pattern of results for this interaction in Table 2 is complex, and its nature is difficult to interpret on the basis of a posteriori comparisons. It seems as if the differences between the small skewing angles $\left(0^{\circ} \pm 15^{\circ}\right)$ are more pronounced for the patterns with $\mathrm{a}$ or an $\mathrm{H}$ axis than for the oblique orientations, whereas the reverse is the case for the large skewing angles $\left( \pm 30^{\circ}\right.$ and $\left.45^{\circ}\right)$.

Number of axes $x$ axis orientation $\times$ skewing angle. Because this highest order interaction was only marginally significant $(p<.08)$, a posteriori comparisons cannot be used to specify the nature of the effect. Nevertheless, some a priori comparisons associated with it are crucial for the main purpose of this experimentnamely, to test the predictions of the model of symmetry detection described above. For example, the detectability of perfect double symmetries was exactly the same as that for skewed quadruple symmetries $\left(d^{\prime}=4.60\right)$. Furthermore, although perfect single symmetries $\left(d^{\prime}=3.40\right)$ were equally as detectable as skewed double ones $\left[d^{\prime}=\right.$ $3.16 ; t(110)<1$, when averaged across axis orientations], perfect single $\mathrm{V}$ symmetry $\left(d^{\prime}=4.44\right)$ was easier than skewed double V-H symmetry $\left[d^{\prime}=3.43 ; t(50)=2.94\right]$.

\section{DISCUSSION}

\section{Summary and Model Predictions}

In general, the effects of multiple axes and skewing interacted as expected. Skewing had an increasingly larger effect on the detectability of symmetry for a decreasing number of axes. In other words, quadruple symmetry was not affected by skewing, whereas double and single symmetry became much harder to detect. Similarly, the third factor manipulated in this experiment (i.e., axis orientation) had no effect for symmetry about four axes, whereas the classically found orientational effects $(\mathrm{V}$ and $\mathrm{H}$ advantage) were replicated for double and (to a lesser extent) single symmetry.

This pattern of results corroborates the principles of our account presented above quite well (orientational effects could be incorporated). In single symmetry, only firstorder relations between symmetric elements remain after skewing (see Figure 2). Although the virtual lines connecting the symmetric elements pairwise still have orientational uniformity and midpoint collinearity, as noticed by Jenkins (1983), the detectability drops considerably. Apparently, the virtual lines connecting nonsymmetric elements are also important. In skewed single symmetry, these are all different, because distances and angles are not invariant under skewing (which is an affine transformation), and the grouping based on them does not confirm the symmetry. In double symmetry, some secondorder relations between pairs of virtual lines (represented by correlation quadrangles) remain after skewing (see Figure 4). More specifically, although the virtual sym- metric trapezoids disappear, some virtual parallelograms are created by skewing the virtual rectangles. As a result of this, the detectability of double symmetry is decreased by skewing, but it remains quite high. In quadruple symmetry, the number of higher order relations remaining after skewing is so high (see Figure 6) that the regularity automatically emerges out of the normal grouping processes based on them. In other words, the symmetry is very salient and easy to detect.

\section{Toward an Invariants-Based Bootstrapping Model}

Although the general ideas behind this framework for symmetry detection seem to be psychologically valid, some specific results are not consonant with the model's implications. As noted above, skewed quadruple symmetry is not easier to detect than perfect double symmetry. Nevertheless, the former has additional virtual parallelograms that are absent in the latter. We have suggested that this is of no additional help, because the number of second-order relations is already high enough. However, alternative explanations are possible. For example, it might also be the case that virtual parallelograms are less strong correlation quadrangles than virtual symmetric trapezoids are. At first sight, this suggestion seems to be corroborated by the fact that skewed double symmetry (with parallelograms) is harder to detect than perfect single symmetry (with symmetric trapezoids), at least about a $V$ axis, but the different numbers involved (i.e., 6 vs. 66) appear to offer a better ground for the latter finding.

The main difference between the two types of correlation quadrangles is the degree in which the grouping can spread along the axis. Symmetric trapezoids facilitate each other because the angles between the parallel sides and the virtual lines connecting them with the next parallel line along the axis are pairwise identical (i.e., the symmetric pair of sides of the trapezoid). Parallelograms do not have this property; the angles between the parallel sides and the virtual lines connecting them with the next parallel line along the axis are not pairwise identical (i.e., the virtual quadrangles connecting the virtual parallelograms are nonsymmetric trapezoids).

Therefore, our account can be focused somewhat more, in that it is not as much the number of correlation quadrangles that determines the saliency of a regularity as it is the degree to which they facilitate or bootstrap each other. This is so because each of the parallel virtual lines plays a role in two subsequent quadrangles along the axis of symmetry, whereas this is not the case in parallelograms (e.g., in skewed double symmetry; see Figure 4).

One approach to specifying this bootstrapping based on invariant second-order relations is to develop a mathematical function expressing the perceptual cost of a particular grouping. This function would incorporate two terms, corresponding with first- and second-order relations. In addition, a stochastic algorithm based on simulated annealing can then be formulated to search for a global optimum minimizing this cost function. Several 
parameters in the cost function and the simulated annealing algorithm could take care that the algorithm converges to a solution more rapidly if more higher order regularities are present and more invariants-based bootstrapping takes place (see the Appendix).

In addition, making use of a computer implementation, one could try to simulate the basic perceptual findings. This work would allow us to propose a real model of a general mechanism for symmetry detection, whereas the study presented here only suggests some of the principles to be incorporated (for an interesting view on the relation between models and mechanisms, see Uttal, 1990). The results of such an analysis will be reported elsewhere (Wagemans, Van Horebeek, Van Gool, \& Swinnen, 1991). In that study, we will also show that similar bootstrapping effects based on invariant second-order relations seem to underly other phenomena of perceptual grouping and regularity detection. For example, processes such as detecting global structure in vector patterns (see, e.g., Caelli \& Dodwell, 1982, 1984; Moraglia, 1989) and solving correspondence problems in stereo (see Akerstrom \& Todd, 1988) and apparent motion (see Werkhoven, Snippe, \& Koenderink, 1990) seem to rely on the same mechanisms.

\section{REFERENCES}

AKerstrom, R. A., \& TodD, J. T. (1988). The perception of stereoscopic transparency. Perception \& Psychophysics, 44, 421-432.

Attneave, F. (1982). Praegnanz and soap bubble systems: A theoretical exploration. In J. Beck (Ed.), Organization and representation in perception (pp. 11-29). Hillsdale, NJ: Erlbaum.

BARLOW, H. B., Reeves, B. C. (1979). The versatility and absolute efficiency of detecting mirror symmetry in random dot displays. $V i$ sion Research, 19, 783-793.

Biederman, I. (1987). Recognition-by-components: A theory of human image understanding. Psychological Review, 94, 115-147.

Bruce, V. G., Morgan, M. J. (1975). Violations of symmetry and repetition in visual patterns. Perception, 4, 239-249.

Caelli, T., \&odwell, P. (1982). The discrimination of structure in vectorgraphs: Local and global effects. Perception \& Psychophysics, 32, 314-326.

Caelu, T., Dodwell, P. (1984). Orientation-position coding and invariance characteristics of pattern discrimination. Perception \& Psychophysics, 36, 159-168.

Carmody, D. P., Nodine, C. F., Locher, P. J. (1977). Global detection of symmetry. Perceptual \& Motor Skills, 45, 1267-1273.

Chipman, S. F., \& Mendelsohn, M. J. (1979). Influence of six types of visual structure on complexity judgments in children and adults. Journal of Experimental Psychology: Human Perception \& Performance, $5,365-378$.

Corbaluis, M. C., \& Roldan, C. E. (1975). Detection of symmetry as a function of angular orientation. Journal of Experimental Psychology: Human Perception \& Performance, 1, 221-230.

Fisher, C. B., BornsteIn, M. H. (1982). Identification of symmetry: Effects of stimulus orientation and head position. Perception \& Psychophysics, 32, 443-448.

Fox, J. (1975). The use of structural diagnostics in recognition. Journal of Experimental Psychology: Human Perception \& Performance, 104, 57-67.

Friedberg, S. A. (1986). Finding the axes of skewed symmetry. Computer Vision, Graphics, \& Image Processing, 34, 138-155.

HAKALAHTI, H. (1983). Estimation of surface orientation using the hypothesis of object symmetry (Tech. Rep. No. TR-1324). College Park, MD: University of Maryland, Center for Automation Research.

HowE, E. JUNG, K. (1987). Judgments of numerosity: Effects of symmetry and goodness in dot pattern arrays. Acta Psychologica, 64, 3-11.

JENKINS, B. (1982). Redundancy in the perception of bilateral symmetry in dot textures. Perception \& Psychophysics, 32, 171-177.

Jenkins, B. (1983). Component processes in the perception of bilaterally symmetric dot textures. Perception \& Psychophysics, 34, 433-440.

JENKINS, B. (1985). Orientational anisotropy in the human visual system. Perception \& Psychophysics, 37, 125-134.

Julesz, B. (1971). Foundations of cyclopean perception. Chicago: University of Chicago Press.

JULESZ, B. (1981). Figure and ground perception in briefly presented isodipole textures. In M. Kubovy \& J. R. Pomerantz (Eds.), Perceptual organization (pp. 27-54). Hillsdale, NJ: Erlbaum.

KANADE, T., \&ENDER, J. R. (1983). Mapping image properties into shape constraints: Skewed symmetry, affine-transformable patterns, and the shape-from-texture paradigm. In J. Beck, B. Hope, \& A. Rosenfeld (Eds.), Human and machine vision (Vol. 1, pp. 237257). New York: Academic Press.

King, M., Meyer, G. E., Tangney, J., \& Biederman, I. (1976). Shape constancy and a perceptual bias towards symmetry. Perception \& Psychophysics, 19, 129-136.

KirkPatrick, S., Gelatt, C. D., \& Vechi, M. P. (1983). Optimization by simulated annealing. Science, 220, 671-680.

LANSKY, P., YAKIMOFF, N., RADIL, T. (1987). On visual orientation of dot patterns. Biological Cybernetics, 56, 389-396.

LANSKy, P., YAkImofF, N., RADIL, T., Mitrani, L. (1989). Errors in estimating the orientation of dot patterns. Perception, 18, 237-242.

Locher, P. J., \& Nodine, C. F. (1973). Influence of stimulus symmetry on visual scanning patterns. Perception \& Psychophysics, 13, 408-412.

LoCher, P. J., Nodine, C. F. (1989). The perceptual value of symmetry. Computers \& Mathematics with Applications, 17, 475-484.

LOCHER, P., WAGEMANs, J. (1991). The effects of element type and spatial grouping on symmetry detection. Manuscript submitted for publication.

LowE, D. G. (1987). Three-dimensional object recognition from twodimensional images. Artificial Intelligence, 31, 355-395.

MACH, E. (1959). The analysis of sensations (C. M. Williams, trans.). New York: Dover. (Original work published 1886)

MoraguA, G. (1989). Display organization and the detection of horizontal line segments. Perception \& Psychophysics, 45, 265-272.

PALmer, S. E. (1985). The role of symmetry in shape perception. Acta Psychologica, 59, 67-90.

Palmer, S. E., Hemenway, K. (1978). Orientation and symmetry: Effects of multiple, rotational, and near symmetries. Joumal of Experimental Psychology: Human Perception \& Performance, 4, 691-702.

PASHLER, H. (1990). Coordinate frame for symmetry detection and object recognition. Joumal of Experimental Psychology: Human Perception \& Performance, 16, 150-163.

ROYER, F. L. (1981). Detection of symmetry. Joumal of Experimental Psychology: Human Perception \& Performance, 7, 1186-1210.

SAARINEN, J. (1988). Detection of mirror symmetry in random dot patterns at different eccentricities. Vision Research, 28, 755-759.

SmITs, J. T. S., \& Vos, P. G. (1986). A model for the perception of curves in dot figures: The role of local salience of "virtual lines." Biological Cybernetics, 54, 407-416.

STEvens, K. A. (1978). Computation of locally parallel structure. Biological Cybernetics, 29, 19-28.

STEVENS, K. A. (1979). Surface perception by local analysis of texture and contour (Tech. Rep. No. 512). Cambridge, MA: MIT Artificial Intelligence Laboratory.

SzIlagyi, P. G., \& BaIRD, J. C. (1977). A quantitative approach to the study of visual symmetry. Perception \& Psychophysics, 22, 287-292.

UTTAL, W. R. (1990). On some two-way barriers between models and mechanisms. Perception \& Psychophysics, 48, 188-203. 
Van Gool, L., Wagemans, J., Vandeneede, I., Oosterlinck, A. (1990). Similarity extraction and modelling. In Proceedings of the Third Intermational Conference on Computer Vision (pp. 530-534). Osaka: IEEE Computer Society.

Wagemans, J., Van Gool, L., d'Ydewalle, G. (1990). Visual search in dot-patterns with bilateral and skewed symmetry. In D. Brogan (Ed.), Visual search (pp. 99-114). Hants, U.K.: Taylor \& Francis.

WAGEMANS, J., VAN Gool, L., D'YDEWALLE, G. (in press). Orientational effects and component processes in symmetry detection. Quarterly Joumal of Experimental Psychology A.

Wagemans, J., Van Horebeek, J., Van Gool, L., \& Swinnen, V. (1991). Transformations versus invariants in regularity detection. Manuscript submitted for publication.

Werkhoven, P., Snippe, H. P., Koenderink, J. J. (1990). Effects of element orientation on apparent motion perception. Perception \& Psychophysics, 47, 509-525.

YodoGAwA, E. (1982). Symmetropy, an entropy-like measure of visual symmetry. Perception \& Psychophysics, 32, 230-240.

\section{NOTES}

1. The concept of virtual lines stems from computational models trying to represent perceptual groupings between elements in a display. For example, Stevens (1978) has introduced the notion to represent a perceived local pairing in Glass patterns. Smits and Vos (1986) have used virtual lines as primitives to detect contours and curves in dot patterns. The interesting property of these representational primitives is based on the fact that they explicitly possess attributes (i.e., length and orientation) that are only implicitly present in the input. However, one does not have to commit oneself to this computational/representational approach to believe in the psychological relevance of physical characteristics in the display (e.g., distances between dots). In this article, we are using the notion of virtual lines as a shorthand to refer to the pairwise relative positions of dots in a dot pattern (i.e., distance and orientation).

2. Our use of first-, second-, and higher order regularity is different from Julesz's classical use of first-, second-, and higher order statistics in texture discrimination (for a clear review, see, e.g., Julesz, 1981). What we mean by first-order is all properties of line segments (i.e., two connected dots), and by second-order all properties of quadrangles (i.e., two connected pairs of line segments). For example, orientational uniformity (Jenkins, 1983) is referred to as a first-order regularity, whereas regularity of relations between two pairs of dots is called second-order.

3. The virtual lines between two parallel virtual lines do not have the same absolute orientation; they only have the same orientation relative to the axis of symmetry. This fact is indicated by pairwise identical angles in the virual quadrangle representing the two pairs of virual lines. To denote the relative nature of this equality, the notion of correlation is used.

4. Of course, the total number of possible virtual quadrangles formed between four elements is much larger still. Here, only the "correct" ones (i.e., those between two symmetric dot pairs) are counted. When our visual system starts to process a dot pattern, it is totally unaware of the "correct" groupings. However, we suppose that, out of the initial stage in which all virtual-line orientations are equally likely, some structure gradually emerges that facilitates the correct connections and/or inhibits all spurious ones. We think our research has something to say about this mechanism, but we postpone our speculations on this point to the Discussion section. All in all, despite the fact that our system might not use all of these 66 virtual quadrangles, they are at least available to be detected or picked up.

5. Again, we do not know whether this additional regularity is used by our mechanism, but it is potential information (see Note 4).

\section{APPENDIX}

In this Appendix, we will briefly mention some of the aspects of our mathematical cost or energy function expressing the lack of regularity or "goodness" in a dot pattern. This function is then minimized with a simulated annealing technique. Let $A$ be
A
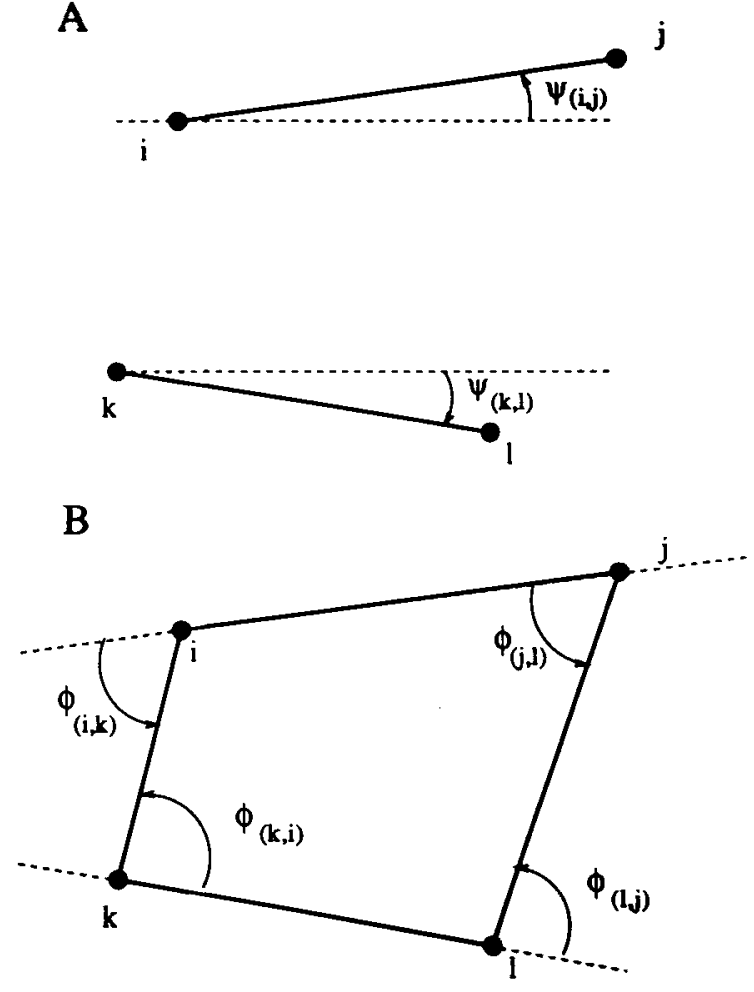

Figure A1. First-order (A) and second-order (B) relations in terms of virtual lines and quadrangles, respectively.

a set of elements representing a pattern of dots $\{i, j, k, \ldots\}$. With every interpretation $x$ - that is, a set of couples of elements in $A$-an energy value $f(x)$ is associated. Although several alternative functions are currently under investigation, all take the relative positioning of point pairs (represented by virtual lines) into account. Previously, models working with relative orientations of such line elements have been proposed in the literature (e.g., Smits \& Vos, 1986; Stevens, 1978). We have referred to such relationships as first-order. Here, we introduce energy functions carrying second-order terms.

Assuming two virtual lines, say $(i, j)$ and $(k, l)$, lying in each other's neighborhood (see Figure A1, panel $A$ ), the firstorder information corresponds to relations between orientations of individual virtual lines-for example, orientation variance or, in our current implementation, orientation differences $\left|\psi_{(i, j)}-\psi_{(k, l)}\right|$. In the different versions of the energy functions, terms of the form $\Sigma\left(e^{\left|\psi_{(i, j)}-\psi_{(k, t)}\right|}-1\right)$ are used, expressing good continuation or parallelism, depending on the stimulus type (e.g., Glass patterns, symmetric patterns, curvilinearity, etc.). The situation for the second-order term is depicted in Figure A1, panel $B$. The angles $\phi$ cannot be defined unless a virtual line pair has been selected (hence, second-order). They are defined relative to the angles $\psi$ and always correspond to the smallest angle enclosed between the virtual lines. The second-order term in our current version takes the form $\sum e^{\left|\phi_{(i, k)}-\phi_{(, i)}\right|+\left|\phi_{(k, i)}-\phi_{(t, j)}\right|}$. For both first- and second-order terms, only restricted neighborhoods of virtual lines are taken into account. In addition to increasing the algorithm's efficiency, this has a clearcut psychological underpinning (see, e.g., Barlow \& Reeves, 1979; Jenkins, 1982). 
The optimization procedure then works as follows. Given a pattern of virtual lines $x_{t}$ at time $t,(1)$ generate a new candidate pattern $y$ for $x_{t+1}$ by adding or removing a virtual line; (2) if the energy value is lower, accept $x_{t+1}=y$; otherwise accept the candidate with a probability $e^{\left[f\left(x_{t}\right)-f(y)\right] / T_{t}}$, with $T_{t}$ a decreasing row converging to 0 .

It is useful to compare the process with the evolution in time of a spinglass system. This is a model used in statistical physics to study the global magnetization characteristics of some materials in terms of the local interaction between the outer electrons of each atom, represented by a vector, called spin (see, e.g., Kirkpatrick, Gelatt, \& Vecchi, 1983). With each couple of points that can be connected, a spin can be associated with two possible states (signs): Active $(+1)$ means that the connection is made; passive (-1) denotes that it is not made. It is essential that the spins can have a range of values between +1 and -1 so that the probability that a spin may change its sign is proportional to its value. For the active spins representing the connections made, this value is proportional to the resistance of the neighborhood on the connection (cf. the first term in the energy function), whereas for the passive spins, this value denotes the potential support of the neighborhood for this connection if it would be made (cf. the second term in the energy function).

At this moment, this idea is implemented in a sequential algorithm. To compute $x_{t+1}$, it is attempted as much as possible to make the most interesting changes first in the generation process. The latter then works as follows: (1) With probability $p$, select two points randomly (in fact, one completely random point, and a second one as the closest neighbor in a region defined by two random corners, one between 0 and $2 \pi$, and the other between $\pi / 8$ and $\pi / 4$ ); (2) with probability $q$, add the most promising connection (i.e., the highest passive spin); and (3) with probability $1-p-q$, remove the most uninteresting connection.

We are currently testing the energy function and the algorithm to investigate its plausibility as a grouping or regularity-detection mechanism. In addition, we will start with a parallel implementation of it by means of transputers.

(Manuscript received August 8, 1990; revision accepted for publication May 21, 1991.) 